\title{
EFFECTS OF DIFFERENT WATER MANAGEMENT METHODS ON SEEDING RATE, PHENOLOGICAL AND YIELDING PROPERTIES OF DIFFERENT RICE CULTIVARS (ORYZA SATIVA L.)
}

\author{
DU, B. $.^{1,2,4 \#-L U O, ~ H . ~ W . ~}{ }^{4 \#}-$ LIU, C. ${ }^{1,2 \#}-$ LEI, C. Y. ${ }^{3}-$ JIANG, S. C. ${ }^{1,2}-$ LOU, Y. G. ${ }^{1,2}-$ XU, Y. \\ Y. ${ }^{1,2}-$ WANG, M. ${ }^{1,2}-$ SHI, L. ${ }^{1,2}-$ XING, D. Y. ${ }^{1,2^{*}}$ \\ ${ }^{1}$ College of Agriculture, Yangtze University, 434025 Jingzhou, PR China \\ ${ }^{2}$ Hubei Cooperative Innovation Center for Major Food Crops, 434025 Jingzhou, PR China \\ ${ }^{3}$ Hubei Provincial Department of Agriculture, 434025 Jingzhou, PR China \\ ${ }^{4}$ Department of Crop Science and Technology, College of Agriculture, South China Agricultural \\ University, 510642 Guangzhou, PR China \\ ${ }^{\#}$ These authors have contributed equally to this work \\ *Corresponding author \\ e-mail: xingdy_2006@126.com \\ (Received $25^{\text {th }}$ Dec 2018; accepted $8^{\text {th }}$ Feb 2019)
}

\begin{abstract}
Flooding management is an effective and environmental-friendly way to reduce weed populations in paddy fields. However, flooding would also cause waterlogging in direct seeding rice production. The present study was conducted to explore the effect of different water management methods at the germination stage on rice growth and yield, with six rice varieties as materials. $0,1,2$, and $3 \mathrm{~cm}$ water layer treatments at germination stage were set and named as I0, I1, I2 and I3, respectively. The result showed that I1 and I2 treatments significantly reduced seedling rate and seedling quality compared to I0. The highest yield was recorded during $\mathrm{I} 0$, while the yield in the case of $\mathrm{I} 3$ treatment was lower than the former but higher than I1 and I2. Similar trends were also recorded in dry matter weight at heading stage and maturity stage.
\end{abstract}

Keywords: rice, water condition, seedling rate, seedling quality, yield, dry matter accumulation

\section{Introduction}

Rice (Oryza sativa L.), as one of the main stable food crops, feeds more than half of the world's population (Ashraf et al., 2017). Pan et al. (2016) demonstrated that more than $60 \%$ of the population live on rice in China while rice makes up $40 \%$ of the country's total grain. It is very important to maintain the rice productivity and improved the yield potential of rice in China.

In Chinese history, transplanted rice production system has been the main rice production modern for a very long time (CabangonTuong and Abdullah, 2002). Traditionally, rice seedlings were transplanted in puddling condition. However, with the severe labor scarcity and low efficiency due to laborious nature of work and lower net returns, transplanted rice production system is becoming more and more unsuitable for Chinese agriculture (Tao et al., 2016). Direct-seeded rice, as a substitutive rice growing technique, has the benefit to reduced production costs, labor and extra efforts for nursery raising, seedling uprooting and transplanting (Liu et al., 2015). Pan et al. (2017) indicated that this new rice production system not only favors earlier crop establishments, but also provides an opportunity to make better use of early season rainfall, and finally leading 
towards increase the grain yield. Nevertheless, direct-seeded rice production system still has many parts no perfect enough.

Water management is an important part in rice production which could greatly affect rice yield and quality. The study of Kong et al. (2017) revealed that short term water management at filling stage was able to improve antioxidant enzyme activity and alleviate the damage from heat stress to rice. Ren et al. (2017) also demonstrated that feebly arid conditions (water potential of - $(15 \pm 5) \mathrm{kPa}$ ) at booting stage could increase 2-acetyl-1pyrroline content in fragrant rice grains significantly. Normally, pre-germinated seeds would be hill-seeded in paddy filed in direct-seeded rice production system. The water management during the germination stage would be important for seedling growth and development. The research of Luo et al. (2018) showed that water layer higher than $4.5 \mathrm{~cm}$ would significantly inhibit the germination and seedling growth and decreased the antioxidant enzyme activity. However, there was no much report about the water management during the germination stage in direct-seeded rice production.

Therefore, present study was conducted in Experimental Research Farm, College of Agriculture, Yangtze University in order to investigate the effect of different water conditions at germination stage on rice growth and development.

\section{Materials and methods}

\section{Seed materials}

Seeds from six rice cultivars, Wandao107, Liangyou168, Fengliangyouxiang-1, Fengliangyou-2, Guofeng-1, Quanyou 801 were used as the experimental materials in this pot experiment. Those rice varieties were all widely grown in Middle China.

\section{Treatment description and growth conditions}

Before sowing, the seeds were soaked in water for $24 \mathrm{~h}$, germinated in manual climatic boxes for another $10 \mathrm{~h}\left(33^{\circ} \mathrm{C}\right)$, then shade-dried. Then, seeds were direct-seeded into soil containing plastic pots $\left(30 \mathrm{~cm}\right.$ in height and $450 \mathrm{~cm}^{2}$ in surface area) and 50 seeds for each pot in triplicate. The different water managements were applied after the sowing and sustained for 25 days. The water layer treatment description is as below:

I0: Maintenance of $0 \mathrm{~cm}$ water layer at soil surface throughout day and night.

I1: Maintenance of $1 \mathrm{~cm}$ water layer at soil surface throughout day and night.

I2: Maintenance of $2 \mathrm{~cm}$ water layer at soil surface throughout day and night.

I3: Maintenance of $3 \mathrm{~cm}$ water layer at soil surface throughout day and night.

15 days after the sowing, the seedling rate was recorded and at 25 days after the sowing, twenty seedlings were collected randomly from each pot for the estimation of dry matter, seedling length, leaf surface area and chlorophyll content. Chlorophyll content was determined by using the SPAD meter 'SPAD-502' (Konica Minolta, Japan). Leaf area of all green leaf blades was measured with Li-Cor area meter (Li-Cor Model 3100, Lincoln, NE). Then, ten seedlings were retained for each pot and every pot in the experiment was remained at $3 \mathrm{~cm}$ water layer at soil surface until harvest. The experiment soil was sandy loam consisting $23.78 \mathrm{~g} / \mathrm{kg}$ organic matter, $1.61 \mathrm{~g} / \mathrm{kg}$ total nitrogen, 78.66 $\mathrm{mg} / \mathrm{kg}$ available nitrogen, $0.87 \mathrm{~g} / \mathrm{kg}$ total phosphorus, $21.79 \mathrm{mg} / \mathrm{kg}$ available phosphorus, $17.64 \mathrm{mg} / \mathrm{kg}$ total potassium, $151.09 \mathrm{mg} / \mathrm{kg}$ available potassium and 6.48 soil $\mathrm{pH}$. The environment of climatic box were set as: $33{ }^{\circ} \mathrm{C}$ days and $27{ }^{\circ} \mathrm{C}$ nights, under $1200 \mathrm{X}$ yellow light intensity and $75 \%$ humidity. 


$$
-4271 \text { - }
$$

\section{Estimation of dry matter accumulation transportation}

At heading stage and maturity, ten rice plants were collected for the determination of dry matter weight. The leaves, stem-sheaths and grains were separated from the plants and dried under the condition of $80^{\circ} \mathrm{C}$ respectively in order to get the estimation of dry matter. Some parameters were calculated as below:

$$
\begin{aligned}
& \text { Transportation rate in leaf }=\frac{\text { leaf weight at heading stage }- \text { leaf weight at maturity stage }}{\text { leaf weight at heading stage }} \\
& \text { Transportation rate in stem }- \text { sheath }=\frac{\text { stem }- \text { sheath weight at heading stage }- \text { stem }- \text { sheath weight at maturity stage }}{\text { grain weight at maturity stage }} \\
& \text { Contribution rate in leaf }=\frac{\text { leaf weight at heading stage }- \text { leaf weight at maturity stage }}{\text { grain weight at heading stage }- \text { grain weight at maturity stage }} \\
& \text { Contribution rate in stem }- \text { sheath }=\frac{\text { stem }- \text { sheath weight at heading stage }- \text { stem }- \text { sheath weight at maturity stage }}{\text { grain weight at heading stage }- \text { grain weight at maturity stage }}
\end{aligned}
$$

\section{Determination of yield and yield related attributes}

At maturity, twenty random rice plants were harvested and threshed manually and sun dried (adjusted to $\sim 14 \%$ moisture contents) to get the grain yield per pot and expressed in grams per plant $\left(\mathrm{g}\right.$ plant $\left.{ }^{-1}\right)$. Grains were separated manually from each panicle to count total number of grains and number of filled grains per panicle. To record 1000-grain weight, six random samples from filled grains were counted, weighed and averaged. Panicle number per pot was measured by counting the panicle numbers of each plant in three pots in each treatment and averaged.

\section{Statistical analysis}

This study was managed as a randomized complete block design with three replicates $(\mathrm{n}=3)$. Data were analyzed on Statistix 8.1 (Analytical Software, Tallahassee, FL, USA) at the probability level of $5 \%(\mathrm{P}<0.05)$. Differences among means were separated by using least significant difference (LSD) test.

\section{Results}

\section{Seedling rate}

As shown in Table 1, different water management affected seedling rate of different rice varieties differently. The highest seedling rate was recorded in I3 condition. Compared to other rice varieties, Wandaol07 had the highest seedling rate which was $67.50 \%$. The lowest seedling rate was recorded in I1 treatment while the seedling rate in Liangyou 168 was lower than other rice varieties.

\section{Seedling quality}

There were some differences in seedling quality of different varieties under different water managements (Table 2). Compared to I0, I1 treatment significantly reduced leaf area and SPAD values while there was no remarkable difference among I0, I2 and I3 in leaf area and SPAD values. The highest plant height and shoot dry weight were both 


$$
-4272 \text { - }
$$

recorded in $\mathrm{I} 3$ and the lowest plant height, root length, shoot dry weight and root dry weight were all recorded in I1.

\section{Dry matter accumulation}

As shown in Table 3, different water managements at germination stage affected dry matter accumulation differently. At heading stage, the trend of leaf dry weight at heading stage was recorded as I0 > I $>$ I $2>$ I1. Similar conditions were also observed in stem-sheath weight and grain weight. At maturity, the highest dry weight of leaf, stem-sheath and grain were all recorded in I0. There was no significant difference between I 2 and I 3 in stem-sheath dry weight and total dry weight. Moreover, the lowest dry matter weight was recorded in I1 at both heading stage and maturity.

Table 1. Differences in survival rates of different varieties under different water layers

\begin{tabular}{|c|c|c|}
\hline Treatment & Variety & Seedling rate $(\%)$ \\
\hline \multirow{7}{*}{ I0 } & Fengliangyou-2 & $26.67 \mathrm{c}$ \\
\hline & Fengliangyouxiang- 1 & $35.00 \mathrm{~b}$ \\
\hline & Liangyou168 & $26.67 \mathrm{c}$ \\
\hline & Wandao107 & $60.00 \mathrm{a}$ \\
\hline & Guofeng-1 & $45.83 \mathrm{a}$ \\
\hline & Quanyou 801 & $35.83 b$ \\
\hline & Mean & 36.95 \\
\hline \multirow{7}{*}{ I1 } & Fengliangyou-2 & $32.50 \mathrm{a}$ \\
\hline & Fengliangyouxiang- 1 & $39.17 \mathrm{a}$ \\
\hline & Liangyou 168 & $18.33 b$ \\
\hline & Wandao107 & $66.67 \mathrm{a}$ \\
\hline & Guofeng-1 & $38.33 \mathrm{a}$ \\
\hline & Quanyou 801 & $22.50 \mathrm{~b}$ \\
\hline & Mean & 31.39 \\
\hline \multirow{7}{*}{ I2 } & Fengliangyou-2 & $34.17 \mathrm{bc}$ \\
\hline & Fengliangyouxiang- 1 & $43.33 \mathrm{ab}$ \\
\hline & Liangyoul68 & $27.50 \mathrm{c}$ \\
\hline & Wandaol07 & $40.83 \mathrm{ab}$ \\
\hline & Guofeng-1 & $49.17 \mathrm{a}$ \\
\hline & Quanyou801 & $33.33 \mathrm{bc}$ \\
\hline & Mean & 38.06 \\
\hline \multirow{7}{*}{$\mathrm{I} 3$} & Fengliangyou-2 & $40.83 b c$ \\
\hline & Fengliangyouxiang- 1 & $48.33 b$ \\
\hline & Liangyou168 & $33.33 \mathrm{c}$ \\
\hline & Wandao107 & $67.50 \mathrm{a}$ \\
\hline & Guofeng-1 & $45.83 b$ \\
\hline & Quanyou 801 & $20.83 d$ \\
\hline & Mean & 42.78 \\
\hline
\end{tabular}

Means in the same column followed by different lower case letters for the same variety differ significantly at $\mathrm{P}<0.05$ by $\mathrm{T}$-test, the same as below 


$$
-4273 \text { - }
$$

Table 2. Differences in seedling quality of different varieties under different water layers

\begin{tabular}{|c|c|c|c|c|c|c|c|}
\hline Treatment & Variety & $\begin{array}{l}\text { Plant } \\
\text { height }\end{array}$ & $\begin{array}{c}\text { Root } \\
\text { length }\end{array}$ & Leaf area & $\begin{array}{c}\text { SPAD } \\
\text { value }\end{array}$ & $\begin{array}{c}\text { Shoot dry } \\
\text { weight }\end{array}$ & $\begin{array}{c}\text { Root dry } \\
\text { weight }\end{array}$ \\
\hline \multirow{7}{*}{ I0 } & Fengliangyou-2 & $32.66 \mathrm{ab}$ & $13.89 \mathrm{~b}$ & $19.43 \mathrm{ab}$ & $35.95 b$ & $0.239 b$ & $0.081 b c$ \\
\hline & Fengliangyouxiang-1 & $36.73 \mathrm{ab}$ & $14.99 \mathrm{ab}$ & $18.15 \mathrm{ab}$ & $39.84 a$ & $0.266 b$ & $0.099 \mathrm{bc}$ \\
\hline & Liangyou168 & $28.70 \mathrm{~b}$ & $14.38 \mathrm{~b}$ & $13.05 \mathrm{~b}$ & 39.23ab & $0.204 \mathrm{~b}$ & $0.073 \mathrm{c}$ \\
\hline & Wandao107 & $37.20 \mathrm{a}$ & $18.17 \mathrm{ab}$ & $19.47 \mathrm{ab}$ & 39.26ab & $0.311 \mathrm{ab}$ & $0.099 \mathrm{bc}$ \\
\hline & Guofeng-1 & $39.62 \mathrm{a}$ & $20.28 \mathrm{a}$ & 18.39ab & $37.76 \mathrm{ab}$ & $0.394 a b$ & $0.124 a b$ \\
\hline & Quanyou 801 & $40.40 \mathrm{a}$ & $15.12 \mathrm{ab}$ & $22.56 \mathrm{a}$ & 39.23ab & $0.462 \mathrm{a}$ & $0.147 \mathrm{a}$ \\
\hline & Mean & $35.89 \mathrm{~b}$ & $16.14 a$ & 18.51a & $38.54 a$ & $0.313 b$ & $0.104 a$ \\
\hline \multirow{7}{*}{ I1 } & Fengliangyou-2 & $19.23 \mathrm{c}$ & $8.14 \mathrm{a}$ & $7.60 \mathrm{c}$ & $31.98 \mathrm{a}$ & $0.067 \mathrm{c}$ & $0.021 \mathrm{~b}$ \\
\hline & Fengliangyouxiang-1 & $19.81 \mathrm{c}$ & $7.59 \mathrm{a}$ & $7.46 \mathrm{c}$ & $32.64 a$ & $0.076 \mathrm{c}$ & $0.019 b$ \\
\hline & Liangyou 168 & $19.26 \mathrm{c}$ & $6.84 \mathrm{a}$ & $3.48 \mathrm{~d}$ & $32.57 \mathrm{a}$ & $0.054 \mathrm{c}$ & $0.012 b$ \\
\hline & Wandao107 & $35.12 \mathrm{ab}$ & $12.61 \mathrm{~b}$ & $11.5 \mathrm{~b}$ & $36.95 \mathrm{a}$ & $0.234 \mathrm{ab}$ & $0.051 b$ \\
\hline & Guofeng-1 & $39.66 \mathrm{a}$ & $13.87 \mathrm{~b}$ & $16.87 \mathrm{a}$ & $34.18 \mathrm{a}$ & $0.320 \mathrm{a}$ & $0.100 \mathrm{a}$ \\
\hline & Quanyou 801 & $26.74 b c$ & $12.51 \mathrm{~b}$ & $10.59 b c$ & $32.57 \mathrm{a}$ & $0.170 \mathrm{bc}$ & $0.047 \mathrm{~b}$ \\
\hline & Mean & $26.64 c$ & $10.26 \mathrm{c}$ & $9.58 \mathrm{~b}$ & $33.48 \mathrm{~b}$ & $0.153 c$ & $0.042 c$ \\
\hline \multirow{7}{*}{$\mathrm{I} 2$} & Fengliangyou-2 & $40.16 a$ & 13.54ab & $21.70 \mathrm{a}$ & $38.3 \mathrm{a}$ & $0.443 \mathrm{a}$ & $0.091 \mathrm{a}$ \\
\hline & Fengliangyouxiang-1 & $38.84 \mathrm{a}$ & $10.47 \mathrm{~b}$ & $18.83 \mathrm{ab}$ & $36.94 \mathrm{a}$ & $0.286 \mathrm{a}$ & $0.062 \mathrm{a}$ \\
\hline & Liangyoul68 & $32.78 \mathrm{a}$ & $11.5 \mathrm{ab}$ & $14.31 b$ & $35.71 \mathrm{a}$ & $0.252 \mathrm{a}$ & $0.056 \mathrm{a}$ \\
\hline & Wandao107 & $39.09 \mathrm{a}$ & $13.4 \mathrm{ab}$ & $17.03 \mathrm{ab}$ & $38.82 \mathrm{a}$ & $0.320 \mathrm{a}$ & $0.067 \mathrm{a}$ \\
\hline & Guofeng-1 & $38.97 \mathrm{a}$ & $16.04 \mathrm{a}$ & $17.28 \mathrm{ab}$ & $35.72 \mathrm{a}$ & $0.334 \mathrm{a}$ & $0.091 \mathrm{a}$ \\
\hline & Quanyou 801 & $41.24 \mathrm{a}$ & $14.16 \mathrm{ab}$ & $21.93 \mathrm{a}$ & $35.71 \mathrm{a}$ & $0.484 \mathrm{a}$ & $0.108 \mathrm{a}$ \\
\hline & Mean & 38.51ab & $13.18 b$ & 18.51a & $36.87 \mathrm{a}$ & $0.353 a$ & $0.079 b$ \\
\hline \multirow{7}{*}{$\mathrm{I} 3$} & Fengliangyou-2 & $40.13 a$ & $13.76 \mathrm{a}$ & $19.11 \mathrm{ab}$ & $39.22 \mathrm{a}$ & $0.439 \mathrm{a}$ & $0.076 \mathrm{a}$ \\
\hline & Fengliangyouxiang-1 & $42.99 a$ & $14.86 \mathrm{a}$ & $24.41 \mathrm{a}$ & $36.94 \mathrm{ab}$ & $0.437 \mathrm{a}$ & $0.076 \mathrm{a}$ \\
\hline & Liangyou168 & $36.06 \mathrm{a}$ & $13.01 \mathrm{a}$ & $20.28 \mathrm{ab}$ & $39.75 \mathrm{a}$ & $0.342 \mathrm{a}$ & $0.060 \mathrm{a}$ \\
\hline & Wandao107 & $44.61 \mathrm{a}$ & $16 \mathrm{a}$ & $23.83 \mathrm{a}$ & $37.07 \mathrm{ab}$ & $0.497 \mathrm{a}$ & $0.098 \mathrm{a}$ \\
\hline & Guofeng-1 & $35.36 \mathrm{a}$ & $15.44 \mathrm{a}$ & $14.10 \mathrm{~b}$ & $32.33 b$ & $0.317 \mathrm{a}$ & $0.069 \mathrm{a}$ \\
\hline & Quanyou 801 & $44.82 \mathrm{a}$ & $15.31 \mathrm{a}$ & $22.4 \mathrm{ab}$ & $39.75 a$ & $0.473 a$ & $0.097 \mathrm{a}$ \\
\hline & Mean & $40.66 a$ & 14.73ab & $20.69 a$ & 37.51a & $0.418 a$ & 0.079ab \\
\hline
\end{tabular}

\section{Matter transportation and contribution during the filling stage}

As shown in Table 4, different water managements during the germination stage affected the matter transportation during the filling stage differently. The leaf transportation rate in I0 was significantly higher than I2 and I3 which were $0.394,0.374$ and 0.372 while there was no significant difference among I0, I1, I2 and I3 in leaf contrition. The lowest stem-sheath transportation rate was recorded in I1 and there was no significant difference among I0, I2 and I3. Furthermore, the highest photosynthesis contribution rate was recorded in $\mathrm{I} 1$ and the lowest was recorded in $\mathrm{I} 0$.

\section{Yield and related attributes}

Different water management during the germination stage affected rice yield by regulating the yield related attributes (Table 5). The highest yield was recorded in I0 treatment as well as panicle number, grain number, seed-setting rate and grain weight. 


$$
-4274 \text { - }
$$

There was no significant difference between I2 and I3 in yield, 1000-grain weight, seedsetting rate and grain number. Furthermore, I1 treatment had the lowest yield due to the lowest panicle number, grain number and 1000-grain weight.

Table 3. Differences in dry matter accumulation of different varieties under different water layers

\begin{tabular}{|c|c|c|c|c|c|c|c|c|c|}
\hline \multirow[b]{2}{*}{ Treatment } & \multirow[b]{2}{*}{ Variety } & \multicolumn{4}{|c|}{ Heading stage } & \multicolumn{4}{|c|}{ Maturity } \\
\hline & & $\begin{array}{c}\text { Leaf dry } \\
\text { weight }\end{array}$ & $\begin{array}{c}\text { Stem-sheath } \\
\text { dry weight }\end{array}$ & $\begin{array}{c}\text { Grain } \\
\text { dry } \\
\text { weight }\end{array}$ & $\begin{array}{c}\text { Total } \\
\text { dry } \\
\text { weight }\end{array}$ & $\begin{array}{c}\text { Leaf dry } \\
\text { weight }\end{array}$ & $\begin{array}{l}\text { Stem-sheath } \\
\text { dry weight }\end{array}$ & $\begin{array}{c}\text { Grain } \\
\text { dry } \\
\text { weight }\end{array}$ & $\begin{array}{c}\text { Total } \\
\text { dry } \\
\text { weight }\end{array}$ \\
\hline \multirow{7}{*}{ I0 } & Fengliangyou-2 & $1.023 \mathrm{~d}$ & $1.830 \mathrm{e}$ & $0.750 \mathrm{e}$ & $3.603 \mathrm{e}$ & $0.633 \mathrm{~cd}$ & $1.723 \mathrm{~d}$ & $2.140 \mathrm{c}$ & $4.497 \mathrm{e}$ \\
\hline & Fengliangyouxiang-1 & $1.112 \mathrm{c}$ & $1.937 \mathrm{~d}$ & $0.770 \mathrm{~d}$ & $3.819 \mathrm{~d}$ & $0.667 \mathrm{c}$ & $1.820 \mathrm{c}$ & $2.203 \mathrm{bc}$ & $4.690 \mathrm{~d}$ \\
\hline & Liangyou168 & $0.826 \mathrm{e}$ & $1.743 \mathrm{f}$ & $0.677 \mathrm{~d}$ & $3.246 \mathrm{f}$ & $0.517 \mathrm{e}$ & $1.620 \mathrm{e}$ & $2.013 \mathrm{~d}$ & $4.150 \mathrm{f}$ \\
\hline & Wandao107 & $1.124 \mathrm{c}$ & $2.127 \mathrm{c}$ & $0.813 \mathrm{c}$ & $4.064 \mathrm{c}$ & $0.620 \mathrm{~d}$ & $2.003 b$ & $2.303 b$ & $4.927 \mathrm{c}$ \\
\hline & Guofeng-1 & $1.203 b$ & $2.237 \mathrm{~b}$ & $0.920 \mathrm{~b}$ & $4.360 \mathrm{~b}$ & $0.740 \mathrm{~b}$ & $2.080 \mathrm{ab}$ & $2.407 \mathrm{ab}$ & $5.227 \mathrm{~b}$ \\
\hline & Quanyou 801 & $1.324 \mathrm{a}$ & $2.330 \mathrm{a}$ & $1.097 \mathrm{a}$ & $4.751 \mathrm{a}$ & $0.830 \mathrm{a}$ & $2.140 \mathrm{a}$ & $2.500 \mathrm{a}$ & $5.470 \mathrm{a}$ \\
\hline & Mean & $1.102 a$ & $2.034 a$ & $0.838 \mathrm{a}$ & 3.974a & $0.668 \mathrm{a}$ & $1.898 \mathrm{a}$ & $2.261 \mathrm{a}$ & $4.827 \mathrm{a}$ \\
\hline \multirow{7}{*}{ I1 } & Fengliangyou-2 & $0.626 \mathrm{e}$ & $1.243 \mathrm{~d}$ & $0.517 \mathrm{~d}$ & $2.386 \mathrm{~d}$ & $0.387 \mathrm{~d}$ & $1.223 \mathrm{~d}$ & $1.523 \mathrm{bc}$ & $3.133 \mathrm{~d}$ \\
\hline & Fengliangyouxiang-1 & $0.631 \mathrm{e}$ & $1.147 \mathrm{e}$ & $0.413 \mathrm{e}$ & $2.191 \mathrm{e}$ & $0.393 d$ & $1.100 \mathrm{e}$ & $1.393 \mathrm{c}$ & $2.887 \mathrm{e}$ \\
\hline & Liangyou168 & $0.670 \mathrm{~d}$ & $1.107 \mathrm{e}$ & $0.400 \mathrm{e}$ & $2.177 \mathrm{e}$ & $0.347 \mathrm{e}$ & $1.047 \mathrm{e}$ & $1.407 \mathrm{c}$ & $2.800 \mathrm{e}$ \\
\hline & Wandao107 & $0.832 b$ & $1.677 \mathrm{~b}$ & $0.620 \mathrm{~b}$ & $3.129 b$ & $0.533 b$ & $1.567 \mathrm{~b}$ & $1.593 \mathrm{~b}$ & $3.693 b$ \\
\hline & Guofeng-1 & $1.246 \mathrm{a}$ & $2.080 \mathrm{a}$ & $0.850 \mathrm{a}$ & $4.176 \mathrm{a}$ & $0.767 \mathrm{a}$ & $2.050 \mathrm{a}$ & $2.093 \mathrm{a}$ & $4.910 \mathrm{a}$ \\
\hline & Quanyou 801 & $0.725 \mathrm{c}$ & $1.530 \mathrm{c}$ & $0.590 \mathrm{c}$ & $2.845 \mathrm{c}$ & $0.487 \mathrm{c}$ & $1.473 \mathrm{c}$ & $1.507 \mathrm{bc}$ & $3.467 \mathrm{c}$ \\
\hline & Mean & $0.788 d$ & $1.464 d$ & $0.565 \mathrm{~d}$ & $2.817 \mathrm{c}$ & $0.486 \mathrm{~d}$ & $1.410 \mathrm{c}$ & $1.586 \mathrm{~d}$ & $3.482 \mathrm{c}$ \\
\hline \multirow{7}{*}{$\mathrm{I} 2$} & Fengliangyou-2 & $0.942 \mathrm{c}$ & $1.777 b$ & $0.727 \mathrm{ab}$ & $3.446 \mathrm{~b}$ & $0.623 a$ & $1.650 \mathrm{ab}$ & $2.107 \mathrm{a}$ & $4.380 \mathrm{ab}$ \\
\hline & Fengliangyouxiang-1 & $1.172 \mathrm{a}$ & $1.327 \mathrm{f}$ & $0.577 \mathrm{c}$ & $3.075 \mathrm{~d}$ & $0.613 \mathrm{a}$ & $1.280 \mathrm{c}$ & $1.703 \mathrm{~d}$ & $3.597 \mathrm{c}$ \\
\hline & Liangyou168 & $0.758 \mathrm{e}$ & $1.473 \mathrm{c}$ & $0.627 \mathrm{c}$ & $2.858 \mathrm{f}$ & $0.510 \mathrm{c}$ & $1.407 \mathrm{c}$ & $1.803 \mathrm{~cd}$ & $3.720 \mathrm{c}$ \\
\hline & Wandao107 & $0.726 \mathrm{e}$ & $1.523 \mathrm{~d}$ & $0.690 \mathrm{~b}$ & $2.939 \mathrm{e}$ & $0.500 \mathrm{c}$ & $1.433 \mathrm{bc}$ & $1.903 \mathrm{bc}$ & $3.837 \mathrm{c}$ \\
\hline & Guofeng-1 & $0.879 \mathrm{~d}$ & $1.637 \mathrm{c}$ & $0.700 \mathrm{~b}$ & $3.216 \mathrm{c}$ & $0.570 \mathrm{~b}$ & $1.537 \mathrm{~b}$ & $2.003 \mathrm{ab}$ & $4.110 \mathrm{bc}$ \\
\hline & Quanyou 801 & $1.136 \mathrm{~b}$ & $1.890 \mathrm{a}$ & $0.787 \mathrm{a}$ & $3.812 \mathrm{a}$ & $0.637 \mathrm{a}$ & $1.747 \mathrm{a}$ & $2.107 \mathrm{a}$ & $4.490 \mathrm{a}$ \\
\hline & Mean & $0.936 c$ & $1.604 \mathrm{c}$ & $0.684 b$ & $3.224 \mathrm{~b}$ & $0.576 b$ & $1.509 \mathrm{~b}$ & $1.938 \mathrm{~b}$ & $4.022 \mathrm{~b}$ \\
\hline \multirow{7}{*}{$\mathrm{I} 3$} & Fengliangyou-2 & $0.940 \mathrm{~d}$ & $1.520 \mathrm{~b}$ & $0.610 \mathrm{~b}$ & $3.070 \mathrm{~d}$ & $0.603 b$ & $1.463 \mathrm{~b}$ & $1.800 \mathrm{~b}$ & 3.867 \\
\hline & Fengliangyouxiang-1 & $1.325 \mathrm{a}$ & $1.420 \mathrm{~b}$ & $0.560 \mathrm{c}$ & $3.305 \mathrm{c}$ & $0.743 \mathrm{a}$ & $1.387 \mathrm{~b}$ & $1.750 \mathrm{~b}$ & 3.88 \\
\hline & Liangyou168 & $0.744 \mathrm{e}$ & $1.307 \mathrm{c}$ & $0.530 \mathrm{c}$ & $2.581 \mathrm{f}$ & $0.503 c$ & $1.223 \mathrm{c}$ & $1.703 \mathrm{~b}$ & 3.43 \\
\hline & Wandao107 & $1.123 b$ & $1.807 \mathrm{a}$ & $0.753 \mathrm{a}$ & $3.683 a$ & $0.657 \mathrm{~b}$ & $1.777 \mathrm{a}$ & $2.103 \mathrm{a}$ & 4.537 \\
\hline & Guofeng-1 & $0.737 \mathrm{e}$ & $1.500 \mathrm{~b}$ & $0.603 \mathrm{~b}$ & $2.840 \mathrm{e}$ & $0.503 \mathrm{c}$ & $1.400 \mathrm{~b}$ & $1.787 \mathrm{~b}$ & 3.69 \\
\hline & Quanyou 801 & $1.024 \mathrm{c}$ & $1.790 \mathrm{a}$ & $0.760 \mathrm{a}$ & $3.574 \mathrm{~b}$ & $0.637 b$ & $1.713 \mathrm{a}$ & $2.117 \mathrm{a}$ & 4.467 \\
\hline & Mean & $0.982 b$ & $1.557 b$ & $0.636 \mathrm{c}$ & 3.175b & $0.608 \mathrm{c}$ & $1.494 b$ & $1.877 \mathrm{c}$ & $3.978 \mathrm{~b}$ \\
\hline
\end{tabular}

Table 4. Differences in matter transportation of different varieties under different water layers

\begin{tabular}{c|c|c|c|c|c|c}
\hline \multirow{2}{*}{ Treatment } & Variety & $\begin{array}{c}\text { Transportation } \\
\text { rate in leaf }\end{array}$ & $\begin{array}{c}\text { Contribution } \\
\text { rate in leaf }\end{array}$ & $\begin{array}{c}\text { Transportation } \\
\text { rate in stem- } \\
\text { sheath }\end{array}$ & $\begin{array}{c}\text { Contribution } \\
\text { rate in stem- } \\
\text { sheath }\end{array}$ & $\begin{array}{c}\text { Contribution } \\
\text { rate of } \\
\text { photosynthesis }\end{array}$ \\
\hline \multirow{3}{*}{ I0 } & Fengliangyou-2 & $0.381 \mathrm{~b}$ & $0.280 \mathrm{~b}$ & $0.058 \mathrm{a}$ & $0.076 \mathrm{~b}$ & $0.643 \mathrm{ab}$ \\
& Fengliangyouxiang-1 & $0.401 \mathrm{~b}$ & $0.312 \mathrm{ab}$ & $0.060 \mathrm{a}$ & $0.082 \mathrm{ab}$ & $0.606 \mathrm{~b}$ \\
& Liangyou168 & $0.375 \mathrm{~b}$ & $0.232 \mathrm{c}$ & $0.071 \mathrm{a}$ & $0.092 \mathrm{ab}$ & $0.676 \mathrm{a}$ \\
& Wandao107 & $0.448 \mathrm{a}$ & $0.338 \mathrm{a}$ & $0.058 \mathrm{a}$ & $0.082 \mathrm{ab}$ & $0.579 \mathrm{bc}$ \\
& Guofeng-1 & $0.384 \mathrm{~b}$ & $0.311 \mathrm{ab}$ & $0.070 \mathrm{a}$ & $0.105 \mathrm{ab}$ & $0.584 \mathrm{~b}$ \\
& Quanyou801 & $0.373 \mathrm{~b}$ & $0.352 \mathrm{a}$ & $0.082 \mathrm{a}$ & $0.135 \mathrm{a}$ & $0.512 \mathrm{c}$ \\
\hline
\end{tabular}




\begin{tabular}{|c|c|c|c|c|c|c|}
\hline \multirow{7}{*}{ I1 } & Fengliangyou-2 & $0.382 b$ & $0.240 \mathrm{~b}$ & $0.016 \mathrm{a}$ & $0.019 \mathrm{~b}$ & $0.742 \mathrm{a}$ \\
\hline & Fengliangyouxiang-1 & $0.377 b$ & $0.244 b$ & $0.041 \mathrm{a}$ & $0.050 \mathrm{ab}$ & $0.707 \mathrm{a}$ \\
\hline & Liangyou168 & $0.483 \mathrm{a}$ & $0.322 \mathrm{a}$ & $0.054 \mathrm{a}$ & $0.061 \mathrm{ab}$ & $0.618 b$ \\
\hline & Wandao107 & $0.359 b c$ & $0.307 \mathrm{bc}$ & $0.066 \mathrm{a}$ & $0.113 \mathrm{a}$ & $0.580 \mathrm{~b}$ \\
\hline & Guofeng-1 & $0.385 b$ & $0.385 b$ & $0.014 \mathrm{a}$ & $0.025 b$ & $0.589 \mathrm{~b}$ \\
\hline & Quanyou 801 & $0.329 \mathrm{c}$ & $0.263 \mathrm{c}$ & $0.037 \mathrm{a}$ & $0.066 \mathrm{ab}$ & $0.671 \mathrm{ab}$ \\
\hline & Mean & 0.386ab & $0.294 a$ & $0.038 b$ & $0.055 b$ & $0.651 \mathrm{a}$ \\
\hline \multirow{7}{*}{ I 2} & Fengliangyou-2 & $0.338 \mathrm{bc}$ & $0.231 \mathrm{c}$ & $0.071 \mathrm{a}$ & $0.092 \mathrm{a}$ & $0.677 b$ \\
\hline & Fengliangyouxiang-1 & $0.477 \mathrm{a}$ & $0.500 \mathrm{a}$ & $0.035 \mathrm{a}$ & $0.041 \mathrm{a}$ & $0.459 \mathrm{a}$ \\
\hline & Liangyoul68 & $0.327 b c$ & $0.211 \mathrm{c}$ & $0.046 \mathrm{a}$ & $0.056 \mathrm{a}$ & $0.733 a$ \\
\hline & Wandao107 & $0.311 \mathrm{c}$ & $0.186 \mathrm{c}$ & $0.059 \mathrm{a}$ & $0.074 \mathrm{a}$ & $0.740 \mathrm{a}$ \\
\hline & Guofeng-1 & $0.352 b$ & $0.238 \mathrm{c}$ & $0.061 \mathrm{a}$ & $0.083 \mathrm{a}$ & $0.679 \mathrm{a}$ \\
\hline & Quanyou 801 & $0.439 \mathrm{a}$ & $0.383 b$ & $0.076 \mathrm{a}$ & $0.114 \mathrm{a}$ & $0.503 b$ \\
\hline & Mean & $0.374 b$ & $0.292 \mathrm{a}$ & 0.058ab & 0.077ab & 0.632ab \\
\hline \multirow{7}{*}{$\mathrm{I} 3$} & Fengliangyou-2 & $0.358 \mathrm{~cd}$ & $0.283 \mathrm{c}$ & $0.037 \mathrm{a}$ & $0.048 \mathrm{a}$ & $0.670 \mathrm{a}$ \\
\hline & Fengliangyouxiang-1 & $0.439 a$ & $0.489 \mathrm{a}$ & $0.024 \mathrm{a}$ & $0.028 \mathrm{a}$ & $0.484 b$ \\
\hline & Liangyou168 & $0.324 d$ & $0.208 \mathrm{~d}$ & $0.064 \mathrm{a}$ & $0.073 \mathrm{a}$ & $0.720 \mathrm{a}$ \\
\hline & Wandao107 & $0.415 \mathrm{ab}$ & $0.347 \mathrm{~b}$ & $0.017 \mathrm{a}$ & $0.023 \mathrm{a}$ & $0.630 \mathrm{a}$ \\
\hline & Guofeng-1 & $0.317 \mathrm{~d}$ & $0.199 \mathrm{~d}$ & $0.066 \mathrm{a}$ & $0.089 \mathrm{a}$ & $0.712 \mathrm{a}$ \\
\hline & Quanyou801 & $0.378 \mathrm{bc}$ & $0.287 \mathrm{c}$ & $0.041 \mathrm{a}$ & $0.064 \mathrm{a}$ & $0.649 \mathrm{a}$ \\
\hline & Mean & 0.372b & $0.302 \mathrm{a}$ & 0.041ab & $0.054 b$ & 0.644ab \\
\hline
\end{tabular}

Table 5. Differences in yield and related attributes of different varieties under different water layers

\begin{tabular}{|c|c|c|c|c|c|c|}
\hline Treatment & Variety & $\begin{array}{c}\text { Panicle } \\
\text { number per } \\
\text { plant }\end{array}$ & $\begin{array}{c}\text { Grains } \\
\text { number per } \\
\text { panicle }\end{array}$ & $\begin{array}{c}\text { Seed-setting } \\
\text { rate }(\%)\end{array}$ & $\begin{array}{c}\text { 1000-grain } \\
\text { weight (g) }\end{array}$ & $\begin{array}{l}\text { Yield (g } \\
\left.\text { plant }^{-1}\right)\end{array}$ \\
\hline \multirow{7}{*}{ I0 } & Fengliangyou-2 & $15.30 \mathrm{ab}$ & $298.55 a$ & $0.93 a$ & $26.61 \mathrm{c}$ & $32.50 \mathrm{c}$ \\
\hline & Fengliangyouxiang-1 & $11.20 \mathrm{~b}$ & $172.19 b$ & $0.90 \mathrm{a}$ & $25.59 \mathrm{~d}$ & $24.65 f$ \\
\hline & Liangyoul68 & $13.50 \mathrm{ab}$ & $225.05 \mathrm{~b}$ & $0.92 \mathrm{a}$ & $27.13 b$ & $27.17 \mathrm{~d}$ \\
\hline & Wandao107 & $17.40 \mathrm{a}$ & $195.43 b$ & $0.93 a$ & $27.11 \mathrm{~b}$ & $40.12 \mathrm{a}$ \\
\hline & Guofeng-1 & $11.10 \mathrm{~b}$ & $204.41 b$ & $0.91 \mathrm{a}$ & $26.46 c$ & $26.67 \mathrm{e}$ \\
\hline & Quanyou801 & $14.10 \mathrm{ab}$ & $190.78 b$ & $0.91 \mathrm{a}$ & $27.61 \mathrm{a}$ & $35.13 \mathrm{a}$ \\
\hline & Mean & $13.80 \mathrm{a}$ & $214.40 \mathrm{a}$ & $0.92 a$ & $26.7506 a$ & $31.04 a$ \\
\hline \multirow{7}{*}{ I1 } & Fengliangyou-2 & $6.90 \mathrm{a}$ & $221.11 \mathrm{a}$ & $0.92 \mathrm{ab}$ & $26.24 \mathrm{c}$ & $12.32 \mathrm{~d}$ \\
\hline & Fengliangyouxiang-1 & $8.00 \mathrm{a}$ & $117.74 d$ & $0.92 \mathrm{ab}$ & $25.49 d$ & $14.36 \mathrm{bc}$ \\
\hline & Liangyoul68 & $8.20 \mathrm{a}$ & $145.29 \mathrm{c}$ & $0.84 \mathrm{c}$ & $26.67 b$ & $13.98 \mathrm{c}$ \\
\hline & Wandao107 & $8.80 \mathrm{a}$ & $158.46 \mathrm{bc}$ & $0.88 b c$ & $26.63 b$ & $18.46 \mathrm{a}$ \\
\hline & Guofeng-1 & $7.00 \mathrm{a}$ & $172.74 \mathrm{~b}$ & $0.9 \mathrm{ab}$ & $25.98 \mathrm{c}$ & $12.70 \mathrm{~d}$ \\
\hline & Quanyou801 & $7.20 \mathrm{a}$ & $177.78 \mathrm{~b}$ & $0.93 \mathrm{a}$ & $27.57 \mathrm{a}$ & $15.15 b$ \\
\hline & Mean & $7.70 \mathrm{c}$ & $165.52 \mathrm{c}$ & $0.9 \mathrm{ab}$ & $26.4317 \mathrm{c}$ & $14.50 \mathrm{c}$ \\
\hline \multirow{7}{*}{$\mathrm{I} 2$} & Fengliangyou-2 & $7.30 \mathrm{a}$ & $250.54 a$ & $0.9 \mathrm{a}$ & $26.20 \mathrm{c}$ & $15.8967 \mathrm{a}$ \\
\hline & Fengliangyouxiang-1 & $7.80 \mathrm{a}$ & $139.59 \mathrm{c}$ & $0.84 \mathrm{a}$ & $25.89 \mathrm{~d}$ & $13.20 \mathrm{~d}$ \\
\hline & Liangyoul68 & $7.40 \mathrm{a}$ & $147.11 b c$ & $0.88 \mathrm{a}$ & $26.94 b$ & $13.39 \mathrm{~d}$ \\
\hline & Wandao107 & $8.00 \mathrm{a}$ & $160.45 b c$ & $0.85 a$ & $26.78 b$ & $15.22 \mathrm{~b}$ \\
\hline & Guofeng-1 & $7.10 \mathrm{a}$ & $186.85 \mathrm{~b}$ & $0.89 \mathrm{a}$ & $26.26 \mathrm{c}$ & $14.15 \mathrm{c}$ \\
\hline & Quanyou 801 & $7.70 \mathrm{a}$ & $180.85 b c$ & $0.86 a$ & $27.39 \mathrm{a}$ & $16.11 \mathrm{a}$ \\
\hline & Mean & $7.60 \mathrm{c}$ & $177.57 b c$ & $0.87 b$ & $26.5772 b$ & 14.66b \\
\hline
\end{tabular}




\begin{tabular}{c|c|c|c|c|c|c}
\hline \multirow{4}{*}{} & Fengliangyou-2 & $13.30 \mathrm{a}$ & $269 \mathrm{a}$ & $0.84 \mathrm{a}$ & $26.43 \mathrm{c}$ & $22.65 \mathrm{a}$ \\
& Fengliangyouxiang-1 & $9.90 \mathrm{ab}$ & $192.15 \mathrm{~b}$ & $0.86 \mathrm{a}$ & $25.29 \mathrm{~d}$ & $15.82 \mathrm{~d}$ \\
& Liangyou168 & $9.90 \mathrm{ab}$ & $147.09 \mathrm{~cd}$ & $0.88 \mathrm{a}$ & $26.93 \mathrm{~b}$ & $15.79 \mathrm{~d}$ \\
& Wandao107 & $8.20 \mathrm{~b}$ & $127.7 \mathrm{~d}$ & $0.89 \mathrm{a}$ & $26.93 \mathrm{~b}$ & $14.80 \mathrm{e}$ \\
& Guofeng-1 & $8.70 \mathrm{~b}$ & $180.22 \mathrm{bc}$ & $0.91 \mathrm{a}$ & $26.25 \mathrm{c}$ & $19.91 \mathrm{c}$ \\
& Quanyou801 & $12.50 \mathrm{ab}$ & $182.39 \mathrm{bc}$ & $0.85 \mathrm{a}$ & $27.73 \mathrm{a}$ & $21.25 \mathrm{~b}$ \\
& Mean & $\mathbf{1 0 . 4 0 b}$ & $\mathbf{1 8 3 . 0 9 b}$ & $\mathbf{0 . 8 7 b}$ & $\mathbf{2 6 . 5 9 2 8 b}$ & $\mathbf{1 8 . 3 7 b}$ \\
\hline
\end{tabular}

\section{Correlation analysis}

As showed in Table 6, the rice yield had significant positive correlation with root length, leaf area, SPAD value and root dry weight of seedling. There also exited a significant positive correlation between panicle number and SPAD value of seedling. Furthermore, the dry matter weight of leaf, stem-sheath and grain at both heading stage and maturity had significant positive correlation with seedling quality (height, root length, leaf area, SPAD value, shoot dry weight and root dry weight).

Table 6. Relationship among seedling quality, yield and dry matter weight

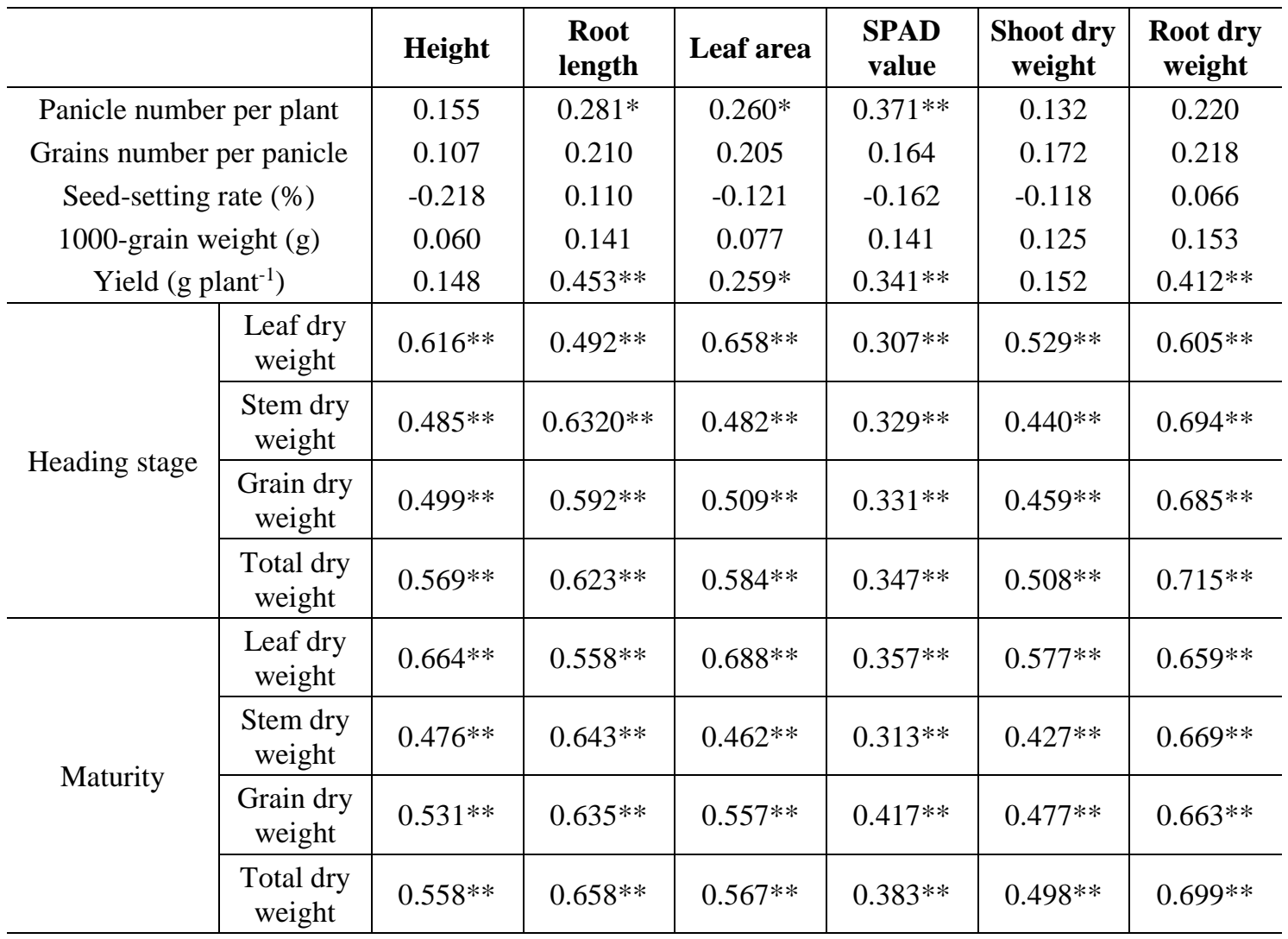

As shown in Table 7, there exited a significant positive correlation between seedsetting rate and stem-sheath dry weight. Both panicle number and yield had significant positive correlation with dry weight of leaf, stem and grain. Moreover, there was a significant positive correlation between grain number and stem-sheath dry weight. 
Table 7. Relationship between dry matter weight at heading stage and yield and yield related attributes

\begin{tabular}{c|c|c|c|c|c}
\hline & $\begin{array}{c}\text { Panicle number } \\
\text { per plant }\end{array}$ & $\begin{array}{c}\text { Grain number } \\
\text { per panicle }\end{array}$ & $\begin{array}{c}\text { Seed-setting } \\
\text { rate }(\%)\end{array}$ & $\begin{array}{c}\text { 1000-grain } \\
\text { weight }(\mathbf{g})\end{array}$ & Yield $\left(\mathbf{g ~ p l a n t}^{-1}\right)$ \\
\hline Leaf dry weight & $0.311^{* *}$ & 0.137 & -0.043 & -0.056 & $0.396^{* *}$ \\
Stem-sheath dry weight & $0.416^{* *}$ & $0.261^{*}$ & $0.233^{*}$ & $0.314^{* *}$ & $0.657^{* *}$ \\
grain dry weight & $0.361^{* *}$ & $0.241^{*}$ & 0.156 & $0.354^{* *}$ & $0.592^{* *}$ \\
Total dry weight & $0.399^{* *}$ & $0.234^{*}$ & 0.139 & 0.223 & $0.603^{* *}$ \\
\hline
\end{tabular}

\section{Discussion}

It is well known that water condition is one of the most important factors affecting the seed germination. Ismail et al. (2009) demonstrated water layer at $10 \mathrm{~cm}$ would greatly inhibit the germination and early seedling growth of rice. The study of Prakash et al. (2016) had evidenced that flooding treatments not only could significantly influenced the survival rate of rice seedling, but also was able to affect all the parameters of seedling quality such as shoot, root and total dry matter production. Present study also showed the similar conditions. Compared to I0, I1 treatment reduced the seedling rate significantly whilst there was no remarkable difference I0 and I3 in seedling rate. The possible reason might be the flooding stress at I3 level could stimulate the mechanisms which were associated with tolerance to flooding for some rice varieties (Zhang et al., 2017). For example, the lowest seedling rates of both Wandaol07 and Fengliangyouxiang-1 were recorded in I2 while there was no significant difference between I0 and I3. Furthermore, we noticed that there was no much significant difference between I0 and I3 in seedling quality. Some parameters (seedling height and shoot dry weight) in I3 were even higher than I0. It might because the $3 \mathrm{~cm}$ water layer conditions would also be suitable for early seedling growth. Similar discovery was reported by Fan et al. (2017) who demonstrated shallow water irrigation during the seedling stage could significant improved seedling quality of fragrant rice.

Seedling quality is one of the factors which could greatly affect the rice yield. Previous study had shown that different seedling age significantly affected yield and economics of hybrid rice by influencing the growth and chlorophyll content (Pramanik and Bera, 2013). The study of Xia et al. (2000) revealed that magnetic treatment was able to up-regulate the rice yield by improving the seedling growth. Normally, it is generally recognized that good quality of rice seedling would be the base for high yield. Our study emphasized this point by showing the significant positive correlation between seedling quality and dry matter accumulation at heading stage and maturity while there exited a significant positive correlation between dry matter production and rice yield. Our founding was also consistent with previous study (Farooq et al., 2006) which stated that seedling establishment is a key part in rice production and good seedling quality could improve the potential of rice yield.

Furthermore, the highest yield was recorded in I0 and the yield in I3 treatment was lower than I0 but higher than I1 and I2. Luo et al. (2018) demonstrated that water layer higher than $3 \mathrm{~cm}$ would inhibit germination and growth of weed and volunteer rice. Thus, $3 \mathrm{~cm}$ water irrigation might be a management which is able to reduce the weed and volunteer without causing a great yield loss. 


\section{Conclusion}

Compared to I0, I1 and I2 treatments significantly reduced seedling rate, seedling quality and the grain yield. I3 treatment was lower than I0 but higher than I1 and I2. Similar trends were also recorded in dry matter weight at heading stage and maturity stage. Therefore, the $3 \mathrm{~cm}$ water irrigation might be considered as a management which is able to reduce the weed and volunteer without causing a great yield loss. More studies should be conducted at the field trials or at molecular level before the real application.

Acknowledgements. This study was supported by Innovation and Application of Key Technologies for Light Simplified Direct Sowing of Rice in the North of the Middle and Lower Reaches of the Yangtze River (2017YFD0301403-06), Research and Demonstration on the Common Key Technologies of Changing Rice into Japonica in Jianghan Plain (2015Z20007) and Key Technological Tackling of Common Characters in Japonica Rice (2015Z20021).

\section{REFERENCES}

[1] Ashraf, U., Kanu, A. S., Deng, Q., Mo, Z., Pan, S., Tian, H., Tang, X. (2017): Lead (Pb) toxicity; physio-biochemical mechanisms, grain yield, quality, and $\mathrm{Pb}$ distribution proportions in scented rice. - Frontiers in Plant Science 8: 259.

[2] Cabangon, R. J., Tuong, T. P., Abdullah, N. B. (2002): Comparing water input and water productivity of transplanted and direct-seeded rice production systems. - Agricultural Water Management 57: 11-31.

[3] Fan, P., Luo, H., Duan, M., Huang, S., Kong, L., Zhong, Z., Zhaowen, M. O., Pan, S. (2017): Effects of shallow water irrigation on morphological and physiological characteristics of direct seeding rice seedling. - Acta Agriculturae Boreali-Sinica 32(5): 185-191.

[4] Farooq, M., Barsa, S. M. A., Wahid, A. (2006): Priming of field-sown rice seed enhances germination, seedling establishment, allometry and yield. - Plant Growth Regulation 49: 285-294.

[5] Ismail, A. M., Ella, G. V. ESVergara, Mackill, D. J. (2009): Mechanisms associated with tolerance to flooding during germination and early seedling growth in rice (Oryza sativa). - Annals of Botany 103: 197-209.

[6] Kong, L., Ashraf, U., Cheng, S., Rao, G., Mo, Z., Tian, H., Pan, S., Tang, X. (2017): Short-term water management at early filling stage improves early-season rice performance under high temperature stress in South China. - European Journal of Agronomy 90: 117-126.

[7] Liu, T. Q., Fan, D. J., Zhang, X. X., Chen, J., Li, C. F., Cao, C. G. (2015): Deep placement of nitrogen fertilizers reduces ammonia volatilization and increases nitrogen utilization efficiency in no-tillage paddy fields in central China. - Field Crops Research 184: 80-90.

[8] Luo, H. W., Du, B., Zheng, A. X., Lai, R. F., You, Z. S., Wang, M., Wang, Z. M., He, L. X., Zhang, T. T., Tang, X. R. (2018): Flooding treatment restrains volunteer rice germination and seedling growth. - Applied Ecology and Environmental Research 16: 7231-7242.

[9] Pan, S., Liu, H., Mo, Z., Patterson, B., Duan, M., Tian, H., Hu, S., Tang, X. (2016): Effects of nitrogen and shading on root morphologies, nutrient accumulation, and photosynthetic parameters in different rice genotypes. - Scientific Reports 6: 32148.

[10] Pan, S., Wen, X., Wang, Z., Ashraf, U., Tian, H., Duan, M., Mo, Z., Fan, P., Tang, X. (2017): Benefits of mechanized deep placement of nitrogen fertilizer in direct-seeded rice in South China. - Field Crops Research 203: 139-149. 


$$
-4279 \text { - }
$$

[11] Prakash, M., Sunilkumar, B., Narayanan, G. S., Gokulakrishnan, J., Anandan, R. (2016): Seed germination and seedling growth of rice varieties as affected by flooding stress. Indian Journal of Agricultural Research 50: 268-272.

[12] Pramanik, K., Bera, A. K. (2013): Effect of seedling age and nitrogen fertilizer on growth, chlorophyll content, yield and economics of hybrid rice (Oryza sativa, L.). International Journal of Agronomy \& Plant Production 4(S): 3489-3499.

[13] Ren, Y., Ashraf, U., He, L. X., Mo, Z. W., Wang, F., Wan, X. C., Kong, H., Ran, X. L., Tang, X. R. (2017): Irrigation and nitrogen management practices affect grain yield and 2-acetyl-1-pyrroline content in aromatic rice. - Applied Ecology and Environmental Research 15: 1447-1460.

[14] Tao, Y., Chen, Q., Peng, S., Wang, W., Nie, L. (2016): Lower global warming potential and higher yield of wet direct-seeded rice in Central China. - Agronomy for Sustainable Development 36: 24.

[15] Xia, L., Liu, X., Yan, Y. I. (2000): Effect of magnetic treatment on seedling growth and yield of rice. - Journal of Jilin Agricultural University 22: 34-37.

[16] Zhang, M., Lu, Q., Wu, W., Niu, X., Wang, C., Feng, Y., Xu, Q., Wang, S., Yuan, X., $\mathrm{Yu}, \mathrm{H}$. (2017): Association mapping reveals novel genetic loci contributing to flooding tolerance during germination in Indica rice. - Frontiers in Plant Science 8. DOI: 10.3389/fpls.2017.00678. 